\title{
A Genetic -based Localization Algorithm for Elderly People in Smart Cities
}

\author{
Zaineb liouane \\ LARATSI - ENIM \\ School, \\ Monastir Tunisia, \\ liouane.zaineb \\ @yahoo.fr
}

\author{
Tayeb Lemlouma \\ IRISA University of \\ Rennes1, \\ Lannion France, \\ Tayeb.Lemlouma \\ @irisa.fr,
}

\author{
Philippe Roose \\ LIUPPA/T2i - UPPA, \\ Anglet France, \\ Philippe.Roose@i \\ utbayonne.univ- \\ pau.fr,
}

\author{
Fréderic Weis \\ IRISA - University \\ of Rennes1, \\ Rennes France. \\ frederic.weis@iri \\ sa.fr
}

\author{
HassaniMessaoud \\ LARATSI - ENIM \\ School, \\ Monastir Tunisia, \\ Hassani.Messaoud \\ @enim.rnu.tn.
}

\begin{abstract}
In the context of aging populations, maintaining persons at home represents one of the solutions seriously considered. In France, one person among three will be over 60 years in 2050, against one among five in 2005 [12]. Many works have investigated the monitoring of elderly peoples inside the institutions or homes (i.e. smart homes) but less in the outside. In this work, we investigate the outdoor monitoring for elderly in the context of smart cities. For this purpose, we focus our research on the problem of localizing the persons when they are outside. We propose an efficient algorithm in terms of rapidity, energy-saving, accuracy and cost. We improve the DV-hop algorithm by using a genetic algorithm in the anchor selection step to efficiently recognize the location of the person and decrease the error rate.
\end{abstract}

\section{Keywords}

Localization, Elderly; Smart Cities; DV-hop algorithm; DV-hop based on GA; prediction.

\section{INTRODUCTION}

Compared to the monitoring of persons in smart homes, in smart cities, the scope of the citizens' activities is large and not limited the set of basic activities of daily living (known as BADL or ADL). Intelligent cities should respond to the needs of the aging population in terms of healthcare, social participation, community support services, leisure and culture in order to guarantee a more friendly and secure environment for this vulnerable category.

In the aim of making the best effort for an independent live style

SAMPLE: Permission to make digital or hard copies of all or part of this work for personal or classroom use is granted without fee provided that copies are not made or distributed for profit or commercial advantage and that copies bear this notice and the full citation on the first page. To copy otherwise, or republish, to post on servers or to redistribute to lists, requires prior specific permission and/or a fee.

Conference'10, Month 1-2, 2010, City, State, Country.

Copyright 2010 ACM 1-58113-000-0/00/0010 ...\$15.00.

DOI: http://dx.doi.org/10.1145/12345.67890 outside the home, the objective is to enable the city to provide an outside persons' monitoring while preserving the ability to perform leisure activities such as sport, shopping, communication with others, etc. Hence, older people and disabled persons can rely on personalized and reliable services that permit a life of quasi-independency with the feeling of being safe thanks to the assistance of appropriate infrastructures that, for instance, can potentially trigger a call for an automatic or human assistance.

In this work, we investigate one of the main issues in supervision and assistance systems for elderly, which is the outside location. Estimating the location outside represents a complex task. Our objective is to ensure a localization service for elderly which should be provided in time and when it's necessary.

Recent research used several protocols and algorithms in order to detect the location of persons, objects, etc. Used approaches can be classified into two categories: range-based and range-free algorithms.

Range-based algorithms such as Received Signal Strength Indicator (RSSI), Time of Arrival, Time Difference of Arrival, require additional hardware and important cost. On the contrary, range-free provide more economical and simpler cost. They do not require information of unknown nodes, however, their results are always less accurate than range-bases. For these reasons, we select the DV-hop algorithm and aim to improve its accuracy.

In the context of smart cities, we consider the DV-hop and genetic algorithms in order to consider the mobility of elderly and provide them a continuous monitoring and assistance. DV-hop is a location algorithm that enables nodes to learn their location without the need of range estimation. Furthermore, it is suitable for sensor positioning due to its cost effectiveness.

DV-Hop algorithm is based on the estimation of the unknown node's location based on the anchor location. Hence, we focus on the improvement of the most important step which is the choice of the anchor node. To do so, propose to investigate the selection of anchors using genetic algorithms to improve the quality of estimation and increase the accuracy of our algorithm.

The particle swarm optimization and fuzzy logic have been recently used with success to evaluate genetic algorithms[15]. 
The paper is organized as follows. In section II, we present the related works. In section III, services of smart cities are described. Section IV is devoted to the wireless body area sensor in the context of a smart city. In section $\mathrm{V}$, we discuss the localization algorithm and focus on the DV-hop one. We describe our proposed algorithm and improvements used to predict the mobility of the elderly outside. Simulations and results, that illustrate the provided model, are described in Section VII.

\section{RELATED WORK}

In the recent years, many research focused the smart environment seen the evolution of the technology in many areas for this reason in this section we interest to study many related works concerned the smart city, the elderly and the algorithm of location.

Skouby et al [10], Propose a detailed study about the increased number of the aging people in the recent year also in the future years,from 2012 the number of elderly increased to almost 810 million it is predicted that more than double in 2050 that's why the necessity of the smart environments to offer a better life of the elderly. In this context monitoring the elderly in smart city touch many problems such as health security, move security, etc.

Chen et al [3], Combine WSN and computer networks to create a multilayer architecture of the smart city based on semantic web technologie. In addition the authors of [3] view the heterogeneity of the information detected from the city the author propose the Dempster Shafer theory for recognizing and reasoning all types of data therefore the author use the multilevel smart city architecture.

Jin et al [6], Presents a framework information to create a smart city through the Internet Of Thing. The authors of [6] detailed precisely the different architecture of IOT such as network-centric IOT , cloud-centric IOT and data-centric IOT also they present the advantage of using this new technology view the variety of information.

In [4] Aamir et al propose a new type of framework to study the challenges of the aged person in the city such as a high disease, limitation of mobility, hearing and visual impairments, for this purpose the authors propose a health and emergency care platform to provide a health state of the disable and aging persons. Thus, for analysis data detected by sensor the author proposes the fuzzy logic theory in order to recognize precisely the health care of the person

Chunping et al [11] Offer a new localization algorithm based on DV-hop in the context of the wireless sensor networks, this author conclude that the DV-hop algorithm based on modifiying hop count achieve a best accuracy localization result. The author of the paper [11] study the crucial problem in the wireless sensor network it is the localization of unknown node, they propose to use a novel algorithm of localization the sensor in the networks. So, the authors propose a new algorithm of DV-HOP based on mass spring model DV-MSO to restraints the location error of DV-hop algorithm, the author show that DV-MSO improve the localization of unknown node with great accuracy.

\section{SMART CITIES}

In our smart cities to ensure a perfect and total surveillance, we propose to combine and link all smart environments such as smart home, smart theater, smart library, smart garden, etc. by an efficient technology where our people find all their needs and offer an independent life, which elderly feels the full responsibility and regain their self confidence. In the aim of the diversity and the variety of data collected from the smart city IOT technology focuses on all aspects of collection, processing and interpretation data.

\subsection{Advantage of IoT}

IOT can be grouped and collected many types of data, such as mobility, health service, energy usage and city safety. Ensure the connection between the city and the monitoring center in the aim to offer the necessary interventions in emergency cases. Therefore, IOT guarantee in the first, the collection of heterogeneous data and to convert them into the common format to become ligible for the user. In the second, the step of the processing data, such as extraction, analysis and fusion the collected data by using an intelligent and efficient method. In the last, IOT provide the data interpretation is based on the representation, reasoning and integration of data by monitoring center. It is an important step enables us to predict and recognize the abnormal event of our person.

\subsection{Protocols of the Internet of Things}

An existing efficient protocol for the Internet of Things (IoT) offer various devices that make them suitable for a large number of applications. In the smart environment, data will be stored in the cloud and may be processed in the cloud, Connections back to the cloud storage, routing of information through wireless and wireline connections to the cloud storage and security are required for this reason a variety of the protocol are used in this area such as : ZigBee, like Bluetooth other available ZigBee profiles, is based on the IEEE802.15.4 protocol, Z-Wave is a low-power RF communications technology that is primarily designed for home automation for products such as lamp controllers and sensors among. A key IP (Internet Protocol) based technology is 6LowPAN (IPv6 Low-power wireless Personal Area Network), SNMP Simple Network Management Protocol used for managing the collections of sensor nodes.etc.

For this purpose it is necessary to use in internet of thing in any smart environment precisely in a heterogeneous environment data.

\section{Wireless Body Area Networks in Smart Cities}

To recognize the location outsideb of the elderly in the aim to offer an independent life, it's necessary to talk the wireless body area networks (WBAN) concerned the aged person[7].

To monitor the health of our patient correctly and dynamically we propose to use a wireless body area network. WBAN technology used for the health monitoring enable us to place a sensor in the body of the person in order to follow the health state like heart rate, level of glucose, temperature of the body, etc.

This type of wireless allow our elderly to move independly outside with a great health security. In the recent years, there are several protocols to aggregate data from the body to the sink and subsequently to the health surveillance center. 


\subsection{The use of WBAN}

It is a smart wireless consists of limited number of nodes deployed in the body of the person. Each sensor concerned a specific disease such as: ECG sensor, glucose sensor, diabetes and cardiovascular sensor more that a sink node responsible to collect all this health data. Thereafter the transmission to the monitoring center is required from the sink.

This type of the wireless help us to monitor the state of health of elderly also helps the person to move with liberty in the city (outside the home) and always stay controlled by their doctor and nurse.

\subsection{WBAN Protocol}

WBAN used to monitor the health care of the people for this purpose in the recent search many authors focused the protocol of WBAN such as Latre et al [7] propose a secure low delay protocol, [9] propose a self-organization protocol for BAN, in addition Nadeem et al [8] propose a new protocol called Stable Increased-Throughput Multi-hop Protocol for Link Efficiency in Wireless Body Area Networks . Those are a routing protocol used to detect dynamically the evolution of the state of health of the elderly [increasing, decreasing or stable] in order to intervene when the situation of the person require it.

\section{Localization of the people}

To achieve the complete freedom of the elderly and to practice all outside daily activities such as shopping, sport, promenades, etc. In this paper, we focus on the crucial challenge it is monitoring the move and the localization of the person dynamically at the real time. In this context, we propose an efficient algorithm to detect the localization of the person based on many criteria linked to the health state such as Forgetting the path/ trajectory, Medication time, fall, Vertigo. All this criteria require an immediate intervention therefore the necessity of a precise identification of person location at time.

The current known location systems are GPS (Global Positioning System) and GSM (Global System for Mobile communications). However, these solutions do not meet the needs of maintaining older people in particular positional accuracy (about 10 meters offset). Today's challenge is to provide a locating system such as "outside" with more accuracy. In other words, the new system in question does not depend on GPS or GSM. In addition, the economic requirement is another important factor to design the kind of system. Therefore, it is expected an embedded system with a minimum cost.

But faced with various constraints such as real-time location, price optimization and energy, computing resource management (storage, processor ..), the width of the communication band, management mobility, conflict management, there's no efficient algorithm. However, to solve these problems, research has produced solutions more optimized and efficient.

Two categories of localization algorithms have been defined: centralized and distributed.

For centralized techniques, all nodes in the network, refer to a base station where all the computation is required. The multidimensional scaling (MDS-Map) and Semi-definite programming (SDP) are two types of treatment usually used in such cases.

To ensure the independence of nodes and avoid these centralized calculations, distributed algorithms have been proposed. Generally, the location is performed by measuring the strength of
Received Signal Indicator (RSSI) of the anchors located at known positions.

But this method may be inaccurate in some circumstances.

Thus, some works use of other mechanisms such as Arrival Time Difference (TDoA) based on ultrasound or audible frequency; these systems have proven accurate location sight but require additional constraints to correct wrong data received. Another device is the Angle Of Arrival (AoA) allowing the angular positioning of the sensors.

These methods are called "Range-based", they can attain an excellent resolution. But more of their dependencies to some unrealistic assumptions about the spread of the signal (if the environment changes), they still require special and expensive equipment. Consequently, localization algorithms called "Rangefree" have been set up as an alternative. First, these algorithms based tags are a distributed algorithm they locate the nodes in the coordinate system of tags. They use different methodologies such as distribution, limiting the edge, the gradient and APIT. These algorithms are based on the number of hops (for example the DVHop, DV-distance, etc.).

Despite the best feature found in the Range-free algorithm such as rapidity, low cost of implementation and not require any special equipment, it is still limited view point the accuracy and certainty about the detection of the location. For this purpose, we think to improve the precision of the elderly location by using the genetic algorithm in the anchors selection phase in order to increase the performance of the DV-hop algorithm (accuracy of the localization) .

\subsection{DV-hop Algorithm}

Is a location algorithm range-free type the highlight is the ability to calculate the position from simple deductions. One of the advantages of this family does not need complex tools to process information.

The method of DV-Hop localization is inspired by distance vector routing protocols to provide an estimate of the mobile position based on the number of hops [14].

Each anchor here is responsible for transmitting a frame containing its position and a field dedicated to count jumps. On receipt of this message, all nodes decide to retransmit or not by comparing the number of hops to the anchor contained in the frame to information stored in memory. If hop-Count is less than the memory contents, the data is updated, the field of the frame is incremented and the retransmitted frame.

Once all the tags have retrieved the position and the number of hops separating them from their homologous, they calculate an average distance of each anchor node (hop-size) with the following formula:

$$
\text { HopSize }_{i}=\frac{\sum_{i \neq j} \sqrt{\left(x_{i}-x_{j}\right)^{2}+\left(y_{i}-y_{j}\right)^{2}}}{\sum_{i \neq j} h_{i j}}
$$

Where $\left(x_{i}, y_{i}\right),\left(x_{j}, y_{j}\right)$ are the coordinates of the anchor $i$ and the anchor $\mathrm{j}, \mathrm{h}_{\mathrm{ij}}$ is the hop count between anchor $\mathrm{i}$ and anchor $\mathrm{j}$.

Then, each node broadcasts the information with its hop-size to the network through the use of controlled flooding. 
The unknown nodes receive information hop-size and keep the first. Simultaneously, they transmit the hop-size their neighboring nodes. After all unknown nodes have received the hop-size of anchor nodes that have the least breaks them, they calculate the distance $d_{i}$ to the anchor nodes based on two factors the hop-size and the minimum number of hop.The formula is as follows:

$$
d_{i}=h_{i d} \times \text { HopSize }_{i}
$$

In the third step, the unknown nodes calculated their position according to the distance from each anchor node which is obtained in the second step. The representation of anchor i coordinates are (xi, yi) and (x,y) are the unknown node coordinates, the following formula shows the distance of each node relative to the anchor node:

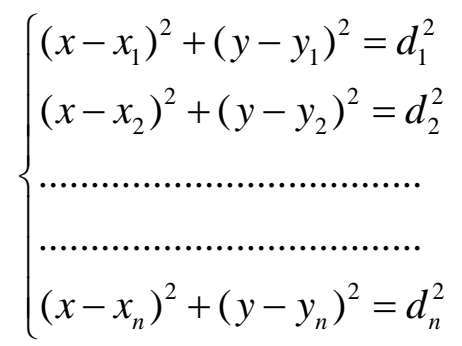

The above formula can be presented as linear equation $\mathrm{AX}=\mathrm{B}$, where

$$
\begin{aligned}
& X=\left(\begin{array}{l}
x \\
y
\end{array}\right), \quad A=2\left[\begin{array}{ll}
x_{n}-x_{1} & y_{n}-y_{1} \\
x_{n}-x_{2} & y_{n}-y_{2} \\
\cdot & \cdot \\
\cdot & \cdot \\
x_{n}-x_{n-1} & y_{n}-y_{n-1}
\end{array}\right]
\end{aligned}
$$

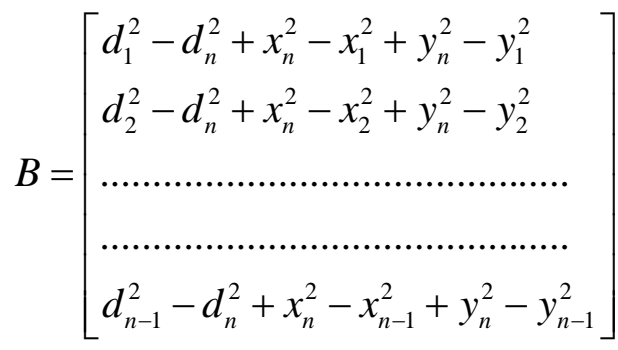

The position of the unknown node is obtained using the least squares method, which may be expressed as follows:

$$
X=\left(A^{T} A\right)^{-1} A^{T} B
$$

\subsection{DV-hop Based on Genetic Algorithm}

Genetic Algorithms (GA) are stochastic search methods inspired from the natural biological evolution. GA operates on a population of potential solutions by applying the principle of survival of the fittest to produce better approximations to a solution [5].
The principle of this algorithm is as follows at each generation. A new set of approximations is created by the process of selecting individuals according to their level of fitness in the problem domain and breeding them together using operators borrowed from natural genetics.

This process leads to the evolution of populations of individuals that are better suited to their environment than the individuals that they were created from, just as in natural adaptation.

Genetic algorithm models operate like natural processes: selection, recombination, mutation and Figure 1 shows the structure of a simple genetic algorithm.

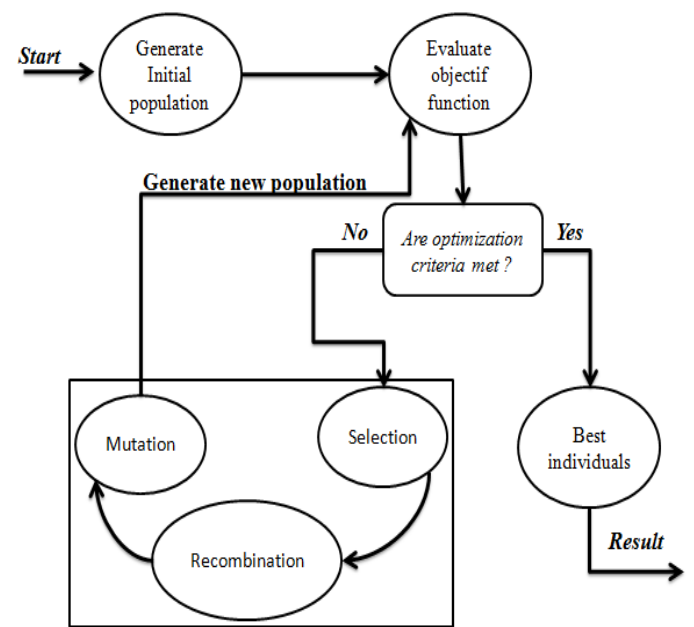

Figure 1. The Principle of the genetic algorithm.

Genetic algorithm work on populations of individuals instead of single individual. At the beginning of the computation a number of individuals (the population) are randomly initialized.

The objective function is then evaluated for these individuals. The first/initial generation is produced. If the optimization criterion is not satisfied, the creation of a new generation starts. Individuals are selected according to their fitness for the production of offspring. Parents are recombined to produce offspring. All offspring will be transferred with a certain probability.

The fitness of the offspring is then computed. The offspring are inserted into the population replacing the parents and producing a new generation. This cycle is then performed until the optimization criterion is achieved. Such a single population genetic algorithm is powerful and performs well on a wide variety of problems.

Suppose that there are $\mathrm{n}+\mathrm{k}$ nodes in a sensor network, which includes $\mathrm{n}$ anchor nodes, whose locations are known, and $\mathrm{k}$ unknown nodes whose locations are unknown. The coordinates of anchor node is $\left(\mathrm{x}_{\mathrm{i}}, \mathrm{y}_{\mathrm{i}}\right),(\mathrm{i}=1,2 \ldots \mathrm{n})$ and the coordinate of unknown node is(x,y) According to the second phase of DV-Hop According to Eq. (3), we get the distance di between an unknown node and the anchor nodes $i$, $(\mathrm{i}=1,2 \ldots . . \mathrm{n})$. 
Because the distance is an estimated value, so there must be an error. Therefore, we proposed an improved DV-Hop based on GA to minimize the error of localization problem in WSN, and the localization problem can be formulated as:

The objective function:

$$
F(x, y)=\operatorname{Min}\left(\sum_{i=1, \ldots . n}\left|\left(x-x_{i}\right)^{2}+\left(y-y_{i}\right)^{2}-d_{i}^{2}\right|\right)
$$

The fitness function as follows:

$$
\text { fitness }(x, y)=\frac{\delta}{F(x, y)}
$$

Where $\delta$ is a positive real coefficient.

The general steps of our proposed algorithm DV-Hop based on GA are summarized as follows:

Table 1. Pseudo-code of the DV-hop based on GA.

\begin{tabular}{ll}
\hline Step1 & $\begin{array}{l}\text { Calculate the minimum hop-count value from each } \\
\text { node to each anchor node; }\end{array}$ \\
\hline Step2 & $\begin{array}{l}\text { According to Eq. (1) estimate the average size for } \\
\text { one hop of each anchor node. }\end{array}$ \\
\hline Step3 & $\begin{array}{l}\text { According to Eq. (2) each unknown node estimates } \\
\text { the distance to the anchor node using the minimum } \\
\text { hop-count value and the average size for one hop. }\end{array}$
\end{tabular}

Step 4 Determine the population feasible region of Each unknown node and generate an initial population in the feasible region;

\begin{tabular}{ll}
\hline Step 5 & Evaluate the fitness of each individual. \\
\hline Step6 & $\begin{array}{l}\text { Three genetic operations (crossover, mutation, and } \\
\text { selection) are performed to generate the next } \\
\text { generation. }\end{array}$
\end{tabular}

\section{This algorithm is repeated till some stopping criterion is met.}

\section{Simulation and Results}

In order to evaluate the location performance of DV-hop based on AG, the experimental simulations are achieved by MATLAB. In this work we will propose that each node in the network represents our patient (elderly) in the city and we will propose that each anchor node is a power pole or a nurse, we can also classify people according to their autonomy 'people has a largest independence can take the role of the anchor'.

The experiment region is a city presented with a square area with the fixed size of $100 \mathrm{~m}^{*} 100 \mathrm{~m}$ in all the experiments.

The structure of our proposed city is composed of a Theater, Library, Cafeteria, Pharmacy, Garden, Gym, Restaurant, Shopping mall and a department consisting of $\mathrm{n}$ smart home $\left(\mathrm{H}_{1} \ldots \ldots \mathrm{H}_{\mathrm{k}}\right)$. Also, our smart city staffed by $\mathrm{k}$ number of elderly equipped by WBANS and $n$ number of anchor node.
The blue nodes represent the elderly residents in the city whose locations are unknown and the red node represent the anchor node whose locations are known as shown in Figure 2.

To evaluate the performance of the DV-hop based on AG we choose initially the number of nodes and the number of anchors.

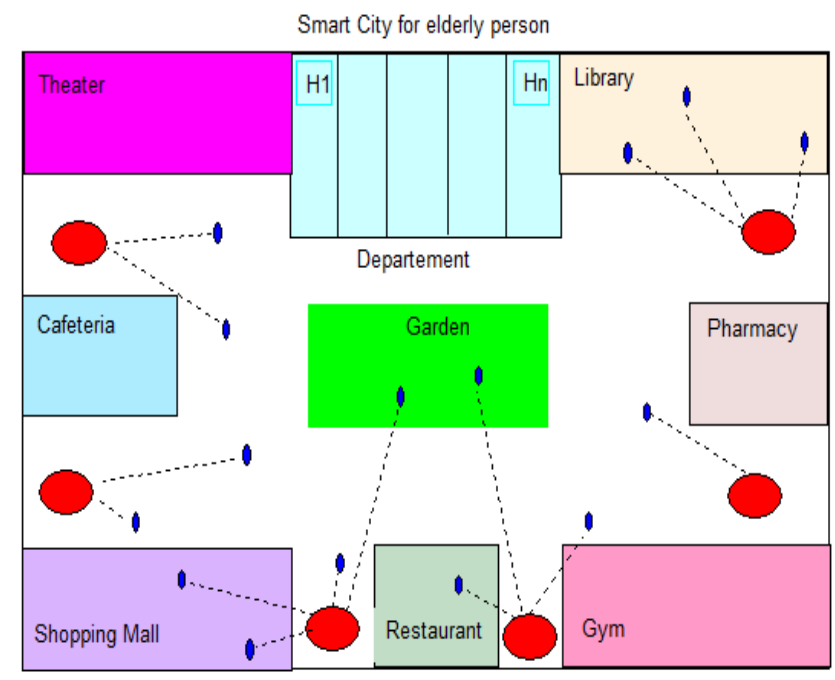

Figure 2. Architecture of our smart city.

In this paper, we execute the results of the location using 30 anchor node and 10 people (unknown node).

Each node is linked to the closest anchor as shown in Figure 2 and dynamically updated when the dimension of the unknown node has changed and subsequently the choose of the new closer anchors should be carried.

Firstly, we study the error accuracy of the location of each unknown node

In this context many emergency cases needed seriously the exact prediction of the location such as :

-The decrease of the health state.

-The elderly Forgetting the path/ trajectory.

-The elderly forgetting Medication time.

-Unexpected event like falling, vertigo, etc.

Therefore, in order to evaluate the performance of our location algorithm we evaluate the error of the location by:

$$
\text { Error }=\sqrt{\left(x_{p}-x_{i}\right)^{2}+\left(y_{p}+y_{i}\right)^{2}}
$$

Where $\left(\mathrm{x}_{\mathrm{p}}, \mathrm{y}_{\mathrm{p}}\right)$ is the predicted coordinate of the unknown node, (xi; yi) is the actual coordinate of the unknown node i.

In order to evaluate the performance of the location, we study in the first the rate of error compared to the number of generations of GA.

Figure 3 shows that the error rate decrease rapidly from 0.1 to 0.03 errors, thereafter decrease to 0.01 in nearly the 2000 generation. 
After simulation of DV-hop based on AG the Error is 0.0185 which it is important results that justify the accuracy of our algorithm.

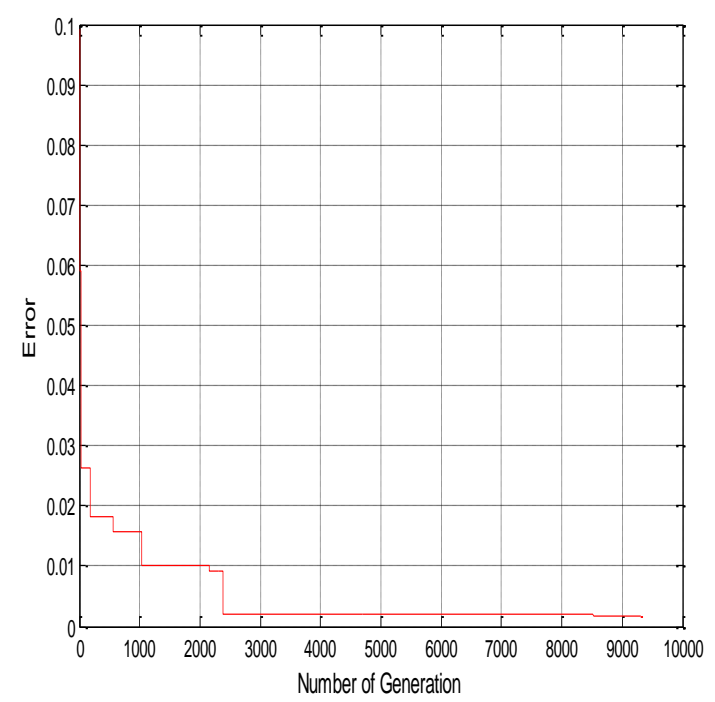

Figure 3. Regression of the error according to the number of generations.

In order to assess the performance of our location algorithm we compare the error location of DV-Hop based on AG and a classic DV-Hop algorithm, Table 2 shows the result of the comparison.

Table 2. Comparison of the prediction error of the localization between the classic DV-hop and the DV-hop based on GA.

\begin{tabular}{ccc}
\hline Algorithm & Classic DV-hop & $\begin{array}{c}\text { DV-hop based } \\
\text { on GA }\end{array}$ \\
\hline Error & 1.1974 & 0.0185 \\
\hline
\end{tabular}

The result shows the performance and the accuracy of the DV-hop based on AG, the location error of the classic DV-hop is 1.1974 but for the DV-hop based on AG is 0.0185 .

The number of anchors has an effect on the accuracy of the location, Figure 4 shows that if we increase the number of anchor node the error of prediction location decrease .

Then we can conclude that to offer an accurate location it is necessary to implement an important number of anchor node, then we should always choose precisely the number of anchors to evolve the performance and to decrease the rate of the accuracy.

\section{CONCLUSION}

Seen the growing number of aging population, it becomes necessary appropriate to propose a smart environment that provide required needs for this vulnerable category of persons either inside a home or in the outside. Elderly and dependent persons require more attention and interest especially in monitoring their activities and actions outside the home where the risk can increase.

In this paper, we were interested to a crucial problem in smart environments that is the outside monitoring with the ability of localizing persons when it is required, for instance in emergency cases. We took advantage of the Internet of Thing (IoT) paradigm for collecting the heterogeneous data that are broadcasted in the city and considered the Wireless Body Area Networks (WBAN) to monitor the health state of the person. To consider the monitoring of the person's mobility, we improved the DV-hop algorithm using the genetic algorithm in the selection of the anchors.

The results confirm that our proposed model provides a better result when compared to the classic DV-hop. We showed that the error of the DV-hop based on GA is very significant (provide a negligible rate of error) . Consequently, the proposed model becomes suitable for health professionals to efficiently monitor the elderly outside and can provide assistance, with a great confidence, in emergency situations.

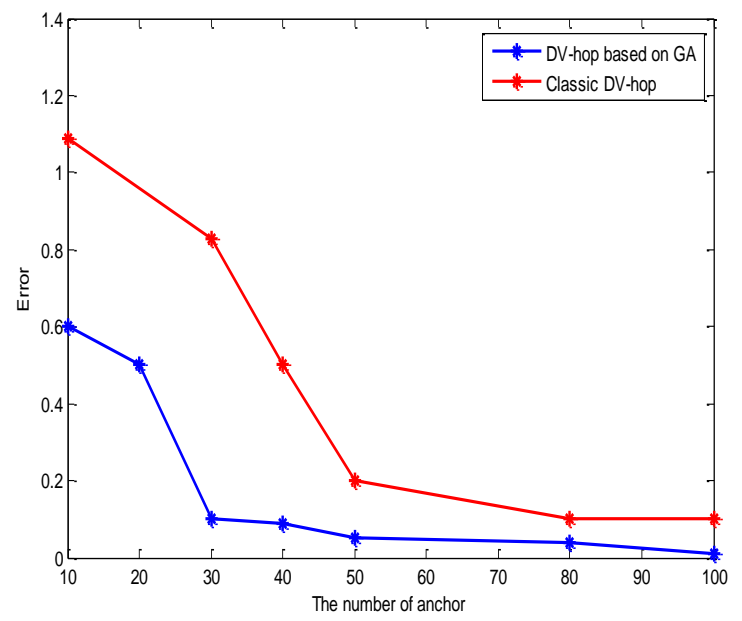

Figure 4. Comparison of the error rate based on the number of anchors between the DV-hop based on GA and the classic DV-hop.

\section{REFERENCES}

[1] ANAGNOSTOPOULOS, Theodoros, KOLOMVATSOS, Kostas, ANAGNOSTOPOULOS, Christos, et al. Assessing dynamic models for high priority waste collection in smart cities. Journal of Systems and Software, 2015, vol. 110, p. 178-192.

[2] GAUR, Aditya, SCOTNEY, Bryan, PARR, Gerard, et al. Smart City Architecture and its Applications Based on IoT. Procedia Computer Science, 2015, vol. 52, p. 10891094.

[3] CHEN, Xiaohui, CHEN, Jinpeng, HE, Jing, et al. An Improved Localization Algorithm for Wireless Sensor Network Based on the Selection of Benchmark Anchor Node. Journal of Networks, 2012, vol. 7, no 6, p. 991-996.

[4] HUSSAIN, Aamir, WENBI, Rao, DA SILVA, Aristides Lopes, et al. Health and emergency-care platform for the elderly and disabled people in the Smart City.Journal of Systems and Software, 2015, vol. 110, p. 253-263. 
[5] JIANG, Chuanjin et FUGEN, Song. Forecasting chaotic time series of exchange rate based on nonlinear autoregressive model. In : Advanced Computer Control (ICACC), 2010 2nd International Conference on. IEEE, 2010. p. 238-241.(4)

[6] JIN, Jiong, GUBBI, Jayavardhana, MARUSIC, Slaven, et al. An information framework for creating a smart city through internet of things. Internet of Things Journal, IEEE, 2014, vol. 1, no 2, p. 112-121.

[7] Latre, Benoit, et al. "A low-delay protocol for multihop wireless body area networks." Mobile and Ubiquitous Systems: Networking and Services, 2007. MobiQuitous 2007. Fourth Annual International Conference on. IEEE, 2007.

[8] NADEEM, Q., JAVAID, N., MOHAMMAD, S. N., et al. SIMPLE: Stable Increased-Throughput Multi-hop Protocol for Link Efficiency in Wireless Body Area Networks. In : 2013 Eighth International Conference on Broadband and Wireless Computing, Communication and Applications.

[9] N. Javaid, N. A. Khan, M. Shakir, M. A. Khan, S. H. Bouk, Z. A. Khan, "Ubiquitous HealthCare in Wireless Body Area Networks-A Survey", J. Basic Appl. Sci. Res. 2013 3(4): 747-759.
[10] SKOUBY, Knud Erik, KIVIMÄKI, Anri, HAUKIPUTO, Lotta, et al. Smart Cities and the Ageing Population. In: The 32nd Meeting of WWRF. 2014.

[11] TU, Chunping. Localization for Wireless Sensor Networks with Improved Dv-Hop and Spring-Model Refinement. International Journal of Digital Content Technology \& its Applications, 2012, vol. 6, no 15.

[12] UNDESA, "Population Division," United Nations Department of Economic and Social Affairs, 2012

[13] Watteyne, Thomas, et al. "Anybody: a self-organization protocol for body area networks." Proceedings of the ICST 2nd international conference on Body area networks. ICST (Institute for Computer Sciences, Social-Informatics and Telecommunications Engineering), 2007.

[14] JIANG, Ming, LI, Yunfei, GE, Yuan, et al. An Advanced DV-hop Localization Algorithm in Wireless Sensor Network. International Journal of Control and Automation, 2015, vol. 8, no 3, p. 405-422.

[15] GONG, Yue-Jiao, LI, Jing-Jing, ZHOU, Yicong, et al. Genetic Learning Particle Swarm Optimization. 2015. 